\title{
First years of single-center experience in liver transplantation
}

\author{
Phu Pham Hong, Nghia Phan Phuoc, Viet Dang Quoc, Dat Le Tien, Thuan Nguyen Duc, Long Tran Cong Duy, Bac Nguyen
}

Hoang

HBP Department, University Medical Center Ho Chi Minh City, Ho Chi Minh City, Vietnam

Background: Liver transplantation is one of the most effective treatments of end-stage benign liver diseases and early hepatocellular carcinoma within Milan criteria. An increasing pool of donor organs plays a major role in the treatment of more patients on the liver transplant waiting list. Currently, liver transplantation has been performed at our center with early satisfactory results. Methods: A prospective cohort study was performed from June 2018 at University Medical Center at Ho Chi Minh City. Selective patients have been satisfied the criteria for liver transplantation.

Results: Overall survival rate after liver transplantation at 6 months, 12 months, 24 months, and 36 months is $82.4 \%, 47.1 \%$, $23.5 \%$, and $5.9 \%$, respectively. The recurrent rate has been not recorded. Two cases died due to severe sepsis. Surgical complications have included hepatic arterial thrombosis $(5.9 \%)$, portal stenosis (11.8\%), middle hepatic venous thrombosis $(23.6 \%)$, biliary leakage (5.9\%), small intestinal perforation (5.9\%), and splenic abscess after splenic arterial ligation (5.9\%). In addition, internal matters have been recorded, such as graft rejection (11.8\%), pneumonitis $(11.8 \%)$, and renal failure (5.9\%). All cases of graft rejection were responded with a high dose of corticosteroids. All cases considered, there were 16 cases of living donor liver transplantation and two cases of deceased donor liver transplantation. One of 16 cases of the living donor was suffered biliary leakage after hepatectomy and treated by endoscopic retrograde cholangiopancreatography with the stent. The mortality rate has not been recorded.

Conclusions: Results of liver transplantation show that satisfactory outcomes of overall survival, complications, morbidity, and mortality. In addition, living donor liver transplant procedure has brought in the safety of living donors and recipients based on the improving techniques of hepatectomy and more ability in the treatment of patients on the waiting list.

Corresponding author: Phu Pham Hong

E-mail:phu.ph@umc.edu.vn

(C) The Korean Society for Transplantation

This is an Open Access article distributed under the terms of the Creative Commons Attribution Non-Commercial License (http://creativecommons.org/licenses/by-nc/4.0/) which permits unrestricted non-commercial use, distribution, and reproduction in any medium, provided the original work is properly cited. 